\title{
Dificultades del pensamiento numérico en los estudiantes de la facultad de ciencias empresariales
}

Omar Castellanos Soriano ${ }^{1}$

\section{Resumen}

Se presenta una investigación sobre dificultades del pensamiento numérico en estudiantes de la Facultad de Ciencias Empresariales, especificamente en la asignatura Matemáticas I, de la Universidad Francisco de Paula Santander. Se ha venido evidenciando un bajo rendimiento académico en la asignatura de Matemática I donde predominan los altos índices de repitencia y deserción universitaria. Es muy frecuente que los docentes se quejen del bajo rendimiento académico y de la mala preparación con que llegan los estudiantes a la universidad, pero no se hacen investigaciones de un carácter formal para identificar las causas de estas dificultades y asi resolverlas haciendo recomendaciones más precisas con el objeto de fortalecer aquellos pensamientos en los que los estudiantes presentan dificultades y así contribuir al mejoramiento del rendimiento académico en el curso inicial de Matemáticas I. El diseño investigativo se centró en un proceso de nivel explicativo, ya que va a permitir identificar las dificultades y conocimientos en el pensamiento numérico de los estudiantes que ingresan a primer semestre en la Facultad de Ciencias Empresariales. Para la recolección de datos se utilizó una prueba diagnóstica en la cual se identifican dos partes, una asociada a la información personal del estudiante (socio-económica) y la otra asociada a factores de tipo académico. Los resultados obtenidos muestran las diferentes dificultades que presentan los estudiantes, y aunque pocos estudiantes tienen buena predisposición hacia el estudio de las matemáticas, en la mayoría los procesos matemáticos no son satisfactorios, lo que es preocupante. Además, no se evidencia que haya diferencias en cuanto la edad ni al género. Finalmente se dan unas recomendaciones con el objeto de empezar a mejorar la situación encontrada.

Palabras Claves: Dificultades, Pensamiento Numérico, Matemáticas I.

\section{Summary}

We present an investigation into difficulties of numerical thinking in students of the Faculty of Business, specifically in the course Mathematics I, University Francisco de Paula Santander. It has been showing poor academic performance in the subject of Mathematics I dominated the high rates of repetition and dropout university. Too often teachers complain of poor academic performance and poor preparation with students coming to college, but did not make a formal research to identify the causes of these problems and resolve them and making more accurate recommendations in order to strengthen

${ }^{1}$ Licenciado en Matemáticas y Computación. Especialista en Estadística Aplicada. Magister en Educación Matemática. Docente del Departamento de Matemáticas y Estadística de La UFPS. e-mail:omarcastellanos69@ufps.edu.co 


\section{Ecomatemático}

those thoughts in which students have difficulties and contribute to improved academic performance in the initial course of Mathematics I. The research design focused on an explanatory level process, as it will help identify the difficulties and numerical thinking knowledge of students entering first semester at the School of Business. For data collection we used a diagnostic test which identifies two parts, one associated with the student's personal information (socio-economic) and other factors associated with academic type. The results show the different difficulties that students have, and although few students have good attitude towards the study of mathematics, most mathematical processes are not satisfactory, which is worrying. Furthermore, no evidence that there are differences in terms of age or gender. Finally some recommendations are given in order to begin to improve the situation encountered.

Keywords: Problems, Numerical Thinking, Math I.

\section{Introducción}

A continuación se presenta la investiga ción sobre dificultades del pensamiento numérico en estudiantes de la Facultad de Ciencias Empresariales, específicamente en la asignatura Matemáticas I, de la Universidad Francisco de Paula Santander.

La investigación surge a raíz de que se ha evidenciado un bajo rendimiento académico en la asignatura de Matemática I donde predominan con altos índices, las dificultades académicas de repitencia y deserción universitaria (SIA-UFPS) [6]. También, es muy frecuente que los docentes se quejen del bajo rendimiento académico y de la mala preparación con que llegan los estudiantes a la universidad, pero no se hacen investigaciones de un carácter formal para identificar las causas de estas dificultades y así encaminar los programas a resolver estas deficiencias haciendo recomendaciones más precisas a las instituciones de secundaria y dentro de la propia universidad en miras a fortalecer aquellos pensamientos en los que los estudiantes presentan dificultades y así contribuir al mejoramiento del rendimiento en el curso inicial de Matemáticas I.

En esta universidad las facultades que más demanda de estudiantes tienen son las de Ingeniería y Ciencias Empresariales, a las cuales pertenece aproximadamente el $65.8 \%$ de los estudiantes ma- triculados, con un índice de deserción del 5.8\% con referencia al total de estudiantes de la universidad (según informe de gestión año 2008) [6]. En donde es necesario que el estudiante que ingresa desarrolle un pensamiento matemático que le permita la comprensión optima de la información y aspectos operativos pero que a su vez permita el desarrollo de un proceso motivador en el plano del aprendizaje para resolver problemas. El estudiante que ingresa a primer semestre es el producto de la educación media, la cual debe garantizar una buena formación en matemáticas según lo reglamentó el Ministerio de Educación Nacional a través de los estándares de educación para matemáticas (Revolución Educativa, Estándares Básicos en Matemáticas Mayo 12 de 2003) [3] los cuales se presentan o se categorizan en pensamientos: El pensamiento numérico y sistemas numéricos, el pensamiento espacial y sistemas geométricos, el pensamiento métrico y sistemas de medidas, el pensamiento aleatorio y sistemas de datos y el pensamiento variacional y sistemas algebraicos y analíticos, los cuales están estrechamente vinculados con los Estándares curriculares y evaluación para la educación matemática del NCTM. [4]

Según lo descrito anteriormente en la Facultad de Ciencias Empresariales de la UFPS se ha venido observando que los estudiantes presentan dificultades en el planteamiento y solución de problemas en la asignatura Matemáticas I y también se evidencia cómo las habilidades del pensamiento numérico desempeña un rol importante dentro de los 


\section{Ecomatemático}

factores que generan este bajo rendimiento. Por tanto la presente investigación toma como referencia el pensamiento numérico dentro del curso de Matemáticas I y así responder los siguientes interrogantes: ¿Qué dificultades en el pensamiento numérico presentan los estudiantes al ingresar al curso de Matemáticas I de la facultad de Ciencias Empresariales de la UFPS? ¿Qué características socioculturales presentan los estudiantes con dificultades en el pensamiento numérico al ingresar al curso de Matemáticas I de la facultad de Ciencias Empresariales de la UFPS? ¿Cuáles son las principales dificultades que presentan los estudiantes al realizar operaciones básicas entre los conjuntos numéricos en la solución de problemas.?

Es así, que para la presente investigación se plantean los siguientes objetivos, un objetivo general que pretende determinar las dificultades alrededor del pensamiento numérico en los estudiantes de primer semestre en la asignatura de Matemáticas I de la facultad de Ciencias Empresariales en la universidad Francisco de Paula Santander y unos objetivos específicos que buscan identificar el nivel de conocimientos y habilidades del pensamiento numérico del estudiante, diagnosticar el nivel de dominio del pensamiento numérico de la población objeto de estudio, caracterizar las dificultades del pensamiento numérico presentes en los estudiantes, realizar una caracterización socio-económico de los estudiantes que presentas dificultades en el pensamiento numérico y finalmente, detectar la opinión del docente frente a las dificultades presentes en los estudiantes en el pensamiento numérico.

\section{Materiales y Métodos}

El diseño investigativo se centró en un proceso de nivel explicativo, ya que va a permitir identificar las dificultades y conocimientos en el pensamiento numérico de los estudiantes que ingresan a primer semestre en la Facultad de Ciencias Empresariales. En el desarrollo del presente proyecto se trabajará con el método inductivo-deductivo. Se partirá de la aplicación de una encuesta (prueba o examen) a los estudiantes que ingresen a primer semestre, la cual determinará en cada caso el nivel de dificultad en el pensamiento numérico.
El diseño investigativo del proyecto se fundamentó en la modalidad de campo donde se recolecta la información mediante una prueba o examen sin manipulación alguna de la variable objeto de estudio.

La presente investigación tiene como población los estudiantes de la Facultad de Ciencias Empresariales de la Universidad Francisco de Paula Santander matriculados en las jornadas diurna y nocturna. Para efectos del proyecto se seleccionó una muestra no probabilística, que está integrada por los estudiantes de primer semestre grupo A de los dos programas sólo en la jornada diurna, aspecto que fue motivado debido a que tras previas averiguaciones se presentan los siguientes factores de ruido:

- En la jornada nocturna tienen acceso personas de diversas edades, en dónde se encuentra que hay estudiantes que se graduaron el año pasado o hace 4, 5 o más años.

- En los grupos A se puede garantizar que no habrán estudiantes repitentes ya que el sistema bloquea el acceso de ellos en este grupo.

- En la jornada nocturna se presenta la situación de que se matriculan estudiantes que ya han culminado algún programa de pregrado en la universidad o en otra institución.

- Las condiciones económicas de los estudiantes de la jornada diurna son distintas a los de la nocturna en términos del pago de matrícula académica ya que es mayor en un $150 \%$ en la jornada nocturna, aspecto que obliga a que quien se matricule en ella deba tener un trabajo estable.

A través de esta investigación se persiguen dos objetivos, inicialmente identificar las dificultades alrededor del pensamiento numérico en los estudiantes del curso de matemáticas I en la Facultad de Ciencias Empresariales, acompañado de una caracterización socio-económica de los estudiantes de dicha facultad. Ello con el fin de buscar ciertas relaciones entre las variables en mención.

Para la recolección de datos se utilizara una prueba diagnóstica en la cual se identifican dos partes, una asociada a la información personal del estudiante (socio-económica) y la otra asociada a factores de tipo académico. En el test hay diversos 


\section{Ecomatemático}

tipos de preguntas, preguntas de asociación, de selección y de selección múltiple con única respuesta.

La confiabilidad y la validez son dos elementos indispensables para la elaboración y aplicación de cualquier instrumento de medición, porque ambas condiciones hacen que los resultados de la investigación puedan ser tomados en serio, en la medida que se acercan más a la representación de las variables por medir.

Confiabilidad. Entendida la confiabilidad de un instrumento como "el grado en que su aplicación repetida al mismo objeto produce iguales resultados" (Hernández y otros, 1998) [2].

Para evaluar la confiabilidad del instrumento y debido a las características del mismo, no es posible aplicar el Alfa de Crombach ya que el test no cuenta con preguntas cuya respuesta sean de escala Licker, para lo cual se propone presentar el instrumento a dos estudiantes que no formen parte de la muestra y en la cual ellos deban indicar la intencionalidad que se persigue en cada ítem.

Validez. Siendo la validez "el grado en que un instrumento realmente mide la(s) variable(s) que pretende medir" (Arias, F) [1], y sabiendo que existen tres tipos de evidencia: de validez, de contenido, de criterio y de contexto.

Para evaluar la validez del instrumento se contará con el aval de dos expertos en el área, los cuales deben cumplir el siguiente perfil: un docente de secundaria con al menos 5 años de experiencia orientando cursos de matemáticas y otro, docente de educación superior que tenga al menos 3 años de experiencia orientando cursos de matemáticas en la facultad de Ciencias Empresariales.

Se realizó un análisis estadístico utilizando el paquete estadístico SSPS y la hoja de Cálculo Microsoft Excel con toda la información recolectada con la finalidad de hacer un análisis descriptivo de la información que permita caracterizar socioeconómica y académicamente los estudiantes de la muestra. Para la presentación de resultados se utilizaron: Cuadros o tabla, y gráficas: gráfica de barras, gráficas de barras compuestas y diagrama circular.

\section{Resultados}

Información personal de los estudiantes. La presencia de mujeres en el primer semestre académico supera el $66 \%$ del tamaño de la población de interés.

Tabla 1. Genero de los estudiantes

\begin{tabular}{lll}
\hline \multirow{2}{*}{ Género } & Descriptivos & \\
\cline { 2 - 3 } & Frecuencia & Porcentaje \\
\hline Femenino & 56 & 65,9 \\
\hline Masculino & 29 & 34,1 \\
\hline Total & $\mathbf{8 5}$ & $\mathbf{1 0 0}$ \\
\hline
\end{tabular}

El rango de edad más frecuente a que están ingresando los estudiantes a este programa académico es entre 17 y 19 años, alcanzando aproximadamente el $78 \%$, lo cual son estudiantes muy jóvenes.

Grafica 1. Frecuencia de los estudiantes por rango de edades

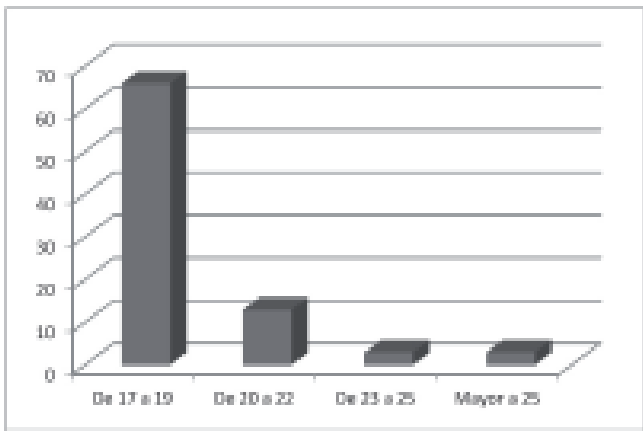

Información académica de los estudiantes previa a su ingreso a la universidad. Aproximadamente el $74 \%$ de los estudiantes de la muestra considerada recibieron título de grado entre los años 2007-2008, aspecto que permite inferir que los conocimientos adquiridos durante el bachillerato aún están fuertes y recientes en sus memorias.

Grafica 2. Año en que se graduó de bachiller

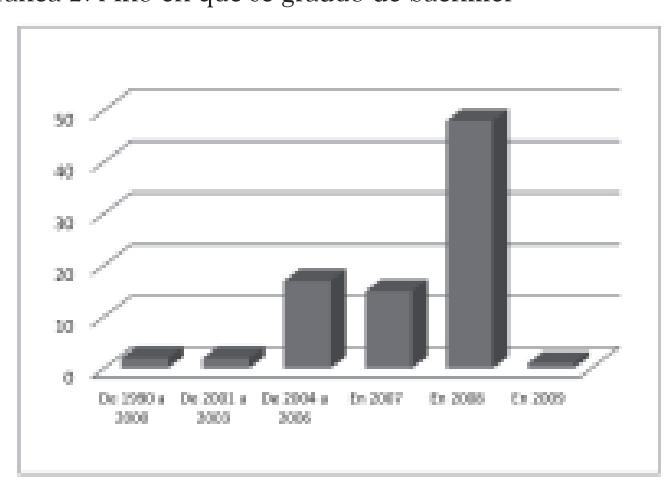




\section{Ecomatemático}

Aproximadamente el $81 \%$ de los estudiantes de la muestra son egresados del sistema educativo público, el cual es subsidiado por el estado y es el fruto de las políticas estatales en función de la educación.

Tabla 2. Tipo de Colegio

\begin{tabular}{lll}
\hline & Descriptivos \\
\cline { 2 - 3 } & Frecuencia & Porcentaje \\
\hline Páblico & 69 & 81,2 \\
\hline Privado & 16 & 18,8 \\
\hline Total & $\mathbf{8 5}$ & $\mathbf{1 0 0}$ \\
\hline
\end{tabular}

La modalidad de bachillerato que predomina en éste programa académico, son aquellos que han desarrollado en su ciclo de educación de media vocacional afinidad hacia la formación académica (profundizan en áreas como matemáticas, español y ciencias naturales) y comercial.

Aproximadamente el 93\% de los estudiantes encuestados son egresados de instituciones educativas con modalidad presencial, aspecto que podría garantizar el tiempo suficiente y necesario para la comprensión y aprehensión de los conceptos matemáticos.

Aproximadamente el $61 \%$ de los estudiantes encuestados obtuvieron en las pruebas ICFES [5] un puntaje que oscila entre 41 y 60 puntos, lo que los ubica dentro de una escala de puntaje medio. Se resalta que el $27 \%$ de ellos no proporcionaron cifras al respecto lo que genera cierto sesgo ya que el porcentaje es representativo.

Tabla 3. Puntaje en las pruebas de estado

\begin{tabular}{lll}
\hline \multirow{2}{*}{ Rango } & Descriptivos \\
\cline { 2 - 3 } & Frecuencia & Porcentaje \\
\hline Entre 30 y 40 & 6 & 7,1 \\
\hline Entre 41 y 50 & 30 & 35,3 \\
\hline Entre 51 y 60 & 22 & 25,9 \\
\hline Entre 61 y 70 & 4 & 4,7 \\
\hline No responden & 23 & 27,0 \\
\hline Total & $\mathbf{8 5}$ & $\mathbf{1 0 0}$
\end{tabular}

Aproximadamente la mitad de los estudiantes encuestados durante sus estudios de educación secundaria perdieron en algún momento la asignatura de matemáticas, aspecto que ratifica de cierta forma el tabú que se ha generado alrededor de esta asignatura en los primeros años de escolaridad.
Aspectos básicos del pensamiento numérico. Aproximadamente el 59\% de los estudiantes encuestados acertaron en asociar las siglas con su respectivo conjunto numérico. Del $41 \%$ de los estudiantes que respondieron de forma incorrecta, el $12 \%$ confunden número racional con número real, el 15\% confunden número irracional con número imaginario y el porcentaje restante asocia de forma aleatoria demostrando desconocimiento del tema, aspecto que resulta preocupante ya que han cursado y aprobado por lo menos once años de educación en los ciclos de primaria y secundaria.

Grafica 3. Relaciona el conjunto numérico con su respectiva sigla

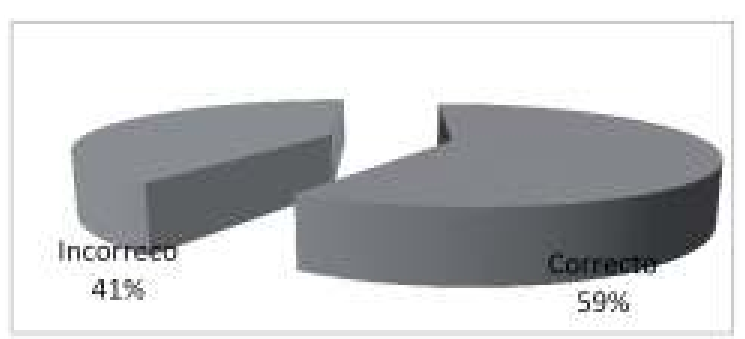

El 100\% de los estudiantes encuestados respondieron de forma incorrecta al ítem donde se le proporcionó a los estudiantes una lista de números y ellos debían indicar con una equis el conjunto numérico al que pertenece cada número., evidenciando gran cantidad de concepciones alrededor de éste tema dentro de las que cabe mencionar:

Tabla 4. Concepciones asociadas a los diferentes conjuntos numéricos

\begin{tabular}{|c|c|}
\hline $\begin{array}{l}\text { Conjunto } \\
\text { Numérice }\end{array}$ & Caracteristica \\
\hline Número Natural & $\begin{array}{l}\text { Número positivo } \\
\text { decimales }\end{array}$ \\
\hline Número Entero & $\begin{array}{l}\text { Número negativo sin cifras } \\
\text { decimales }\end{array}$ \\
\hline Número Racional & $\begin{array}{l}\text { Todo nủmero expresado como } \\
\text { fracción o con cifras decimales }\end{array}$ \\
\hline Número Irracional & $\begin{array}{l}\text { Número que se origina de sacar raiz } \\
\text { cuadrada a cualquier número sin } \\
\text { importar si es positivo o negativo }\end{array}$ \\
\hline
\end{tabular}

Adicional a lo anteriormente mencionado, se suma que no establecen relaciones de contenencia entre los diferentes conjuntos, es decir, creen que un número pertenece exclusivamente a un conjunto numérico, desconociendo situaciones como el caso de los números naturales que están contenidos en los enteros, o los números enteros contenidos dentro de los reales, entre otros. 


\section{Ecomatemático}

Lo exhibido por los estudiantes en los ítems 1 y 2, evidencia el desconocimiento total de las características básicas asociadas a los diversos conjuntos numéricos, luego se plantea el siguiente interrogante: iSi los estudiantes desconocen los conceptos básicos del pensamiento numérico, es posible que los utilicen de forma correcta?, pues este interrogante se intentará responder a partir del análisis de los siguientes numerales dentro del segundo instrumento.

Aspectos operativos del pensamiento numérico. El 84\% de los estudiantes de la muestra se evidencian errores en la solución de los polinomios aritméticos con números enteros, de los cuales el 35\% utilizaron de forma incorrecta la ley de signos, el $23 \%$ se equivocaron al realizar restas o al multiplicar números y el porcentaje restante, simplemente no realizaron proceso alguno.

Grafica 4. Aspectos operativos del pensamiento numérico

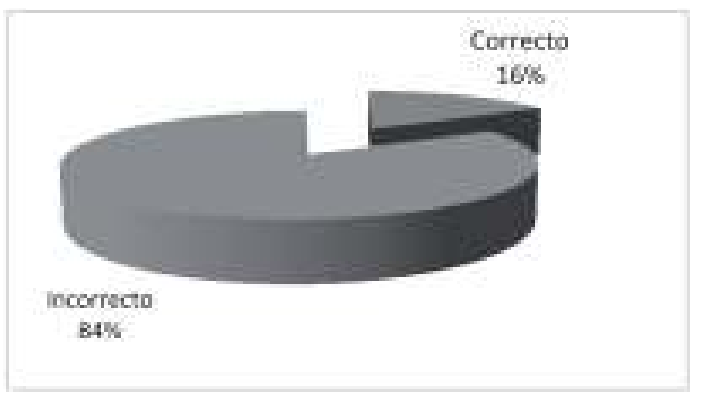

El 88\% de los estudiantes de la muestra se evidencian errores en la solución de los polinomios aritméticos con números racionales, de los cuales el $29 \%$ realizan suman de fracciones de forma inadecuada (numerador con numerador y denominador con denominador), el $17 \%$ al realizar la operación e intentar simplificar lo hacen de forma incorrecta como efecto de que se les ha olvidado las tablas de multiplicar, el 5\% no saben el algoritmo de la división y el porcentaje restante, simplemente no realizan proceso alguno.

Lo exhibido por los estudiantes, evidencia un bajo nivel de dominio sobre el manejo de operaciones básicas en los conjuntos numéricos de los enteros y de los números racionales, lo que debería ser una fortaleza ya que dada la estructura curricular de matemáticas en nuestro sistema educativo, los estudiantes están tratando estos temas desde la educación primaria a partir de cuarto grado, y el nivel de los polinomios propuestos es bastante básico.
Problemas de aplicación del pensamiento numérico. El porcentaje de desacierto en esta pregunta alcanza el $61 \%$, evidenciando cierta dificultad en la interpretación de enunciados ya que de las respuestas incorrectas elegidas, el 11\% optaron por aquella dónde sólo se realizaba la división y el 32\% eligieron aquella dónde sólo se realizó la resta; si a esto se le suma el $17 \%$ de no respuesta genera un alto porcentaje de dificultad en este tipo de enunciados.

Grafico 5. Problemas de aplicación del pensamiento numérico.

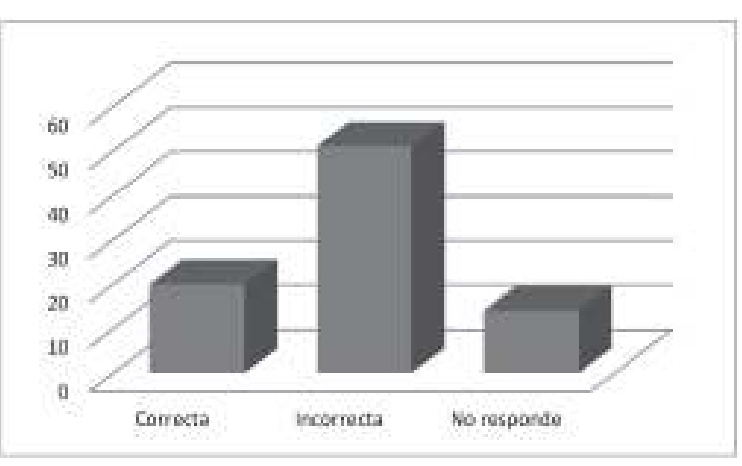

Se evidencia el aumento considerable en el índice de respuestas incorrectas, que aproximadamente llega al $80 \%$, ello en contrate con la disminución del porcentaje de no respuesta.

Se evidencia el alto porcentaje de respuestas incorrectas las cuales alcanzan aproximadamente el $66 \%$, de los cuales el $50 \%$ de ellos eligieron la opción de respuesta equivocada que establecía que los tres recipientes ofrecían igual capacidad, a lo cual se llegaba apoyándose en el concepto de área y no de volumen.

En esta pregunta el porcentaje de desacierto alcanza aproximadamente el 53\%, acompañado de un $35 \%$ de no respuesta. Dentro de las opciones de respuesta incorrectas el 50\% de los estudiantes eligieron la opción que afirmaba que todas las dimensiones dadas proporcionaban la misma área.

Al observar las respuestas dadas por los estudiantes en esta sección del instrumento se puede afirmar que existe gran dificultad en los estudiantes al momento de resolver problemas en contexto, situación que se agudiza cuando los enunciados se proporcionan a partir del registro gráfico, implicando del estudiante un esfuerzo adicional que es el interpretar las gráficas. 


\section{Ecomatemático}

\section{Conclusiones y Recomendaciones}

A continuación se realiza un pequeño resumen de los hallazgos obtenidos en la investigación, lo que permite llegar a generalizar conclusiones y poder hacer algunas recomendaciones acerca de las dificultades de pensamiento numérico en el curso de matemáticas I.

Estas conclusiones están basadas en los hallazgos más representativos de los test aplicados a los estudiantes de Matemáticas I de la Facultad de Ciencias Empresariales y las recomendaciones darán una visión a futuras investigaciones que profundicen o complementen la presente investigación.

Tabla 5. Resumen de Hallazgos

\begin{tabular}{|c|c|c|c|}
\hline DIMENSION & INDICADOR & ITEMS & $\begin{array}{l}\text { CARACTERISTICA DE LA } \\
\text { DEBILIDAD }\end{array}$ \\
\hline \multirow{3}{*}{$\begin{array}{l}\text { Conjuntos } \\
\text { Numéricos }\end{array}$} & $\begin{array}{l}\text { Identifica la sigla } \\
\text { asociada a cada } \\
\text { conjunto numérico }\end{array}$ & $\begin{array}{l}\text { Item } \\
N^{\circ} 1\end{array}$ & $\begin{array}{l}\text { Se confunde un número } \\
\text { racional con un número } \\
\text { real, un número irracio- } \\
\text { nal con un número ima- } \\
\text { ginario. }\end{array}$ \\
\hline & $\begin{array}{l}\text { Identifica las carac- } \\
\text { terísticas propias de } \\
\text { cada conjunto. }\end{array}$ & $\begin{array}{l}\text { Ítem } \\
\text { N²2 }\end{array}$ & $\begin{array}{l}\text { No se establece relación } \\
\text { de contenencia entre } \\
\text { los diferentes conjuntos } \\
\text { numéricos y descono- } \\
\text { cimiento de las carac- } \\
\text { terísticas básicas de los } \\
\text { conjuntos numéricos. }\end{array}$ \\
\hline & $\begin{array}{l}\text { Establece las rela- } \\
\text { ciones de contenen- } \\
\text { cia entre conjuntos } \\
\text { numéricos. }\end{array}$ & $\begin{array}{l}\text { Ítem } \\
\mathrm{N}^{\circ} 3\end{array}$ & $\begin{array}{l}\text { Se evidencian errores } \\
\text { en la solución de poli- } \\
\text { nomios aritméticos, di- } \\
\text { ficultad marcada en las } \\
\text { operaciones básicas. }\end{array}$ \\
\hline \multirow{3}{*}{ Operaciones } & $\begin{array}{l}\text { Realiza operaciones } \\
\text { básicas con núme- } \\
\text { ros enteros. }\end{array}$ & $\begin{array}{l}\text { Ítem } \\
N^{\circ} 4\end{array}$ & $\begin{array}{l}\text { Dificultad al realizar ope- } \\
\text { raciones con racionales, } \\
\text { simplificación de expre- } \\
\text { siones y algoritmos para } \\
\text { la evaluaciones de ope- } \\
\text { raciones básicas. }\end{array}$ \\
\hline & $\begin{array}{l}\text { Realiza operaciones } \\
\text { básicas con núme- } \\
\text { ros Racionales. }\end{array}$ & $\begin{array}{l}\text { Ítem } \\
\text { N5 }\end{array}$ & $\begin{array}{l}\text { Dificultad en la interpre- } \\
\text { tación de enunciados, no } \\
\text { hay claridad en el tipo de } \\
\text { operación correcta para } \\
\text { la búsqueda de la solu- } \\
\text { ción. }\end{array}$ \\
\hline & $\begin{array}{l}\text { Realiza operaciones } \\
\text { básicas con núme- } \\
\text { ros Reales. }\end{array}$ & $\begin{array}{l}\text { Ítem } \\
\mathrm{N}^{\circ} 6\end{array}$ & $\begin{array}{l}\text { No hay claridad para } \\
\text { interpretar situaciones } \\
\text { del lenguaje cotidiano } \\
\text { que genera una opera- } \\
\text { ción básica con números } \\
\text { reales. }\end{array}$ \\
\hline \multirow{2}{*}{$\begin{array}{l}\text { Aplicación } \\
\text { de los } \\
\text { conjuntos } \\
\text { numéricos }\end{array}$} & $\begin{array}{l}\text { Resuelve problemas } \\
\text { en contexto a partir } \\
\text { del lenguaje coti- } \\
\text { diano. }\end{array}$ & $\begin{array}{l}\text { Ítem } \\
N^{\circ} 7\end{array}$ & $\begin{array}{l}\text { Confunden el concepto } \\
\text { de área con el de volu- } \\
\text { men. }\end{array}$ \\
\hline & $\begin{array}{l}\text { Resuelve problemas } \\
\text { en contexto a partir } \\
\text { de representacio- } \\
\text { nes gráficas. }\end{array}$ & $\begin{array}{l}\text { Ítem } \\
\mathrm{N}^{\circ} 8\end{array}$ & $\begin{array}{l}\text { Se dificulta desarrollar } \\
\text { problemas en contexto }\end{array}$ \\
\hline
\end{tabular}

\section{Referencias Bibliográficas}

[1] Arias, F. (2006). El Proyecto de Investigación: Introducción a la Metodología. Científica. (5a. Ed). Caracas, Venezuela: EPISTEME

[2] Hernández, S., Fernández, C. y Baptista, P. (1998). Metodología de la Investigación. (2 ed). México: McGraw-Hill.

[3] Ministerio de Educación Nacional - República de Colombia. (2003). La revolución educativa: estándares básicos de matemáticas y lenguaje educación básica y media. Bogotá.

[4] National Council Teachers Mathematics. (1991). Estándares curriculares y evaluación para la educación matemática. S.A.E.M. Thales.

[5] ICFES. [Página web] www. Icfes.gov.co. Consultada el 13 de julio de 2009 .

[6] Universidad Francisco de Paula Santander. Sistema de Información Académica. [Base de datos]. Consultada el 30 de agosto de 2009 